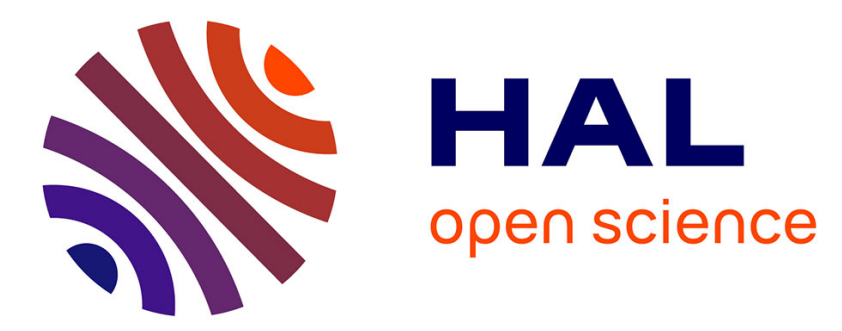

\title{
A Wave Superposition Method Based on Monopole Sources with Unique Solution for All Wave Numbers
}

\author{
Alexandre Leblanc, Ros Kiri Ing, Antoine Lavie
}

\section{To cite this version:}

Alexandre Leblanc, Ros Kiri Ing, Antoine Lavie. A Wave Superposition Method Based on Monopole Sources with Unique Solution for All Wave Numbers. Acta Acustica united with Acustica, 2010, 96, pp.125 - 130. 10.3813/aaa.918263 . hal-03207837

\section{HAL Id: hal-03207837 \\ https://hal-univ-artois.archives-ouvertes.fr/hal-03207837}

Submitted on 26 Apr 2021

HAL is a multi-disciplinary open access archive for the deposit and dissemination of scientific research documents, whether they are published or not. The documents may come from teaching and research institutions in France or abroad, or from public or private research centers.
L'archive ouverte pluridisciplinaire HAL, est destinée au dépôt et à la diffusion de documents scientifiques de niveau recherche, publiés ou non, émanant des établissements d'enseignement et de recherche français ou étrangers, des laboratoires publics ou privés. 


\title{
A Wave Superposition Method Based on Monopole Sources with Unique Solution for All Wave Numbers
}

\author{
Alexandre Leblanc ${ }^{1)}$, Ros Kiri Ing ${ }^{1) *}$, Antoine Lavie ${ }^{2)}$ \\ 1) Institut Langevin, ESPCI ParisTech, CNRS UMR 7587, Laboratoire Ondes et Acoustique, 10 rue Vauquelin, \\ 75231 Paris Cedex 05, France. ale.leblanc@gmail.com \\ 2) Univ Lille Nord de France, 59000 Lille, France. UArtois, LAMTI, 62400 Béthune, France
}

\begin{abstract}
Summary
A simple solution for the uniqueness problem of the wave superposition method is proposed in this paper. Many authors have pointed out the discrete set of wavenumbers for which the solution of the underlying integral equations is not unique. So far, the usual solutions are theoretically sophisticated and/or numerically disadvantageous. Here, by adding some sources interior to the virtual surface defined by the wave superposition method, the uniqueness problem can be easily removed with low computational effort. Furthermore, dealing with simple monopoles, this method is well-suited for practical applications.
\end{abstract}

PACS no. 43.20.Rz, 43.40.Rj

\section{Introduction}

The boundary integral equations have been thoroughly studied since the 1960 s for analyzing acoustic radiation and scattering from any arbitrary vibrating body. Applied in an infinite domain, Boundary Element Methods (BEMs) are recognized to be more efficient than Finite Element Methods (FEMs), especially through the reduction of the computational dimension of the problem by one. Furthermore, the Sommerfeld condition is automatically satisfied so that the external domain doesn't need to be bounded. The two major drawbacks of the BEMs lie on numerical computation of the Helmholtz integral equation: the treatment of singular integrals and the nonuniqueness solutions at the eigenfrequencies of the corresponding Dirichlet interior problem. The latter problem is of primary influence on BEM results as fictitious eigenvalues density raises quickly with the frequency. Several approachs have been proposed in order to eliminate the non-uniqueness problem. Three typical ones are the CHIEF method presented by Shenck [1], the BurtonMiller method [2] and the Null-field method [3]. The CHIEF method is based on the Helmholtz integral equation on the surface of the radiating body combined with Helmholtz equation for some interior points. The resulting overdetermined set of equations is usually solved by the least-square approach. The Burton-Miller approach,

Received 10 June 2009 ,

accepted 20 October 2009.

* Also at Denis Diderot University (Paris 7) inspired by Panich formulation [4], forms a linear combination of the Helmholtz boundary integral equation and its normal derivative, providing also a valid solution at any frequency but leading to hyper-singular integrals which can be handle following Guiggani algorithm [5, 6]. The Null-field method, which is combined with the Helmholtz integral surface equation [7], uses the known bilinear expansion for the simple wave source to express the integral relation in a set of equations leading to a unique solution.

Another BEM related approach is the Wave Superposition Method (WSM): a simple numerical technique for free-field radiation and scattering problems, originally formalized by Koopmann [8]. The principle of the WSM can be found in the literature under various denominations as the Method of Fundamental Solutions [9] (MFS), the sources simulation method [10], the auxiliary [11] or equivalent [12] sources method. The main aspects of the most usual methods dealing with sound source reconstruction can be found in a recent review [13]. While different in theoretical aspects, all theses formulations are based on the idea that the real body is substituted by a set of elementary sources located in its interior, so the global acoustic field of interest can be approximated by the sum of the fields due to each sources. Being a straightforward direct method, the WSM offers several advantages. First, computational cost is lower than BEM or FEM. Second, the WSM constitutes an efficient and economical simulation technique for practical applications. As a setback to the simplicity of this principle, the determination of the source strengths leads to numerical and analytical difficulties, extensively discussed over the past two decades. Following the first WSM stability analysis [14], numerous 
alternatives have emerged to overcome one of the major drawbacks of the method: the non-uniqueness of solutions at fictitious resonant frequencies [15]. To solve this issue, one elegant solution is to use a hybrid combination of single and double layer potentials [16] as the Burton-Miller formulation. This method still one of the most reliable [17] even for high frequencies, but the use of such an analytical sophistication may compromise practical applications. Aside the optimal sources placements studies [18, 14], numerical schemes as Tikhonov regulation or Singular Values Decomposition (SVD) were also successfully applied $[19,20]$ to improve WSM results, but also somewhat overlooked as a solution for the non-uniqueness problem. Indeed, only a severe regulation or high SVD truncation allows to smoothen enough the resonant responses. Thus, and as a counterpart of this significant change in sources to field positions dependencies, the WSM error rises to an unacceptable level. Another attempt was to add an appropriate damping to the virtual source system by the mean of a complex radius vector [21]. Though this technique can insure the unique solutions for all wave numbers if enough damping is introduced, choosing the complex radius vector is a difficult task, as too much damping will deteriorate the method accuracy.

The aim of this paper is to present a merged formulation of the two conceptions behind the superposition methods. The first is the discretization of the interior form of Helmholtz integral equation and the second is the search of fundamental solutions for randomly placed sources. The performance of the proposed method at critical wave numbers and its application in accurate free-field radiation prediction are then evaluated through numerical experiments.

\section{General theory}

Figure 1 shows the geometry of the radiation problem. the well-known Helmholtz differential equation governs the propagation in finite fluid domain $\Sigma$,

$$
\nabla p(\boldsymbol{r})+k^{2} p(\boldsymbol{r})=0 \quad \boldsymbol{r} \in \Sigma,
$$

where $p$ is the pressure at point $\boldsymbol{r}$ in the surrounded fluid $\Sigma$ (assuming an implicit $e^{-j \omega t}$ dependence). This equation is associated with Neumann boundary condition on the surface $\Gamma$,

$$
\frac{\partial p(\boldsymbol{r})}{\partial \boldsymbol{n}}=\mathrm{j} \omega \rho v_{n}(\boldsymbol{r}) \quad \boldsymbol{r} \in \Gamma,
$$

in which $\partial \boldsymbol{n}$ denotes normal differentiation at the point $\boldsymbol{r}$ in the direction from the interior region $\Omega$ toward $\Gamma, v_{n}(\boldsymbol{r})$ is the normal component of the velocity at point $\boldsymbol{r}$ and $\rho$ is the fluid density modulus. The pressure must also satisfy the radiation condition

$$
\lim _{R \rightarrow \infty} \int_{S_{R}}\left|\frac{\partial p(\boldsymbol{r})}{\partial \boldsymbol{r}_{\mathbf{0}}}-\mathrm{j} k p(\boldsymbol{r})\right|_{r=R}^{2} \mathrm{~d} S=0,
$$

where $\boldsymbol{r}_{\mathbf{0}}$ is the radial distance from the origin of coordinates and $S_{R}$ is a sphere of radius $R$ centered at the origin and surrounding $\boldsymbol{r}$ and $\Gamma$.

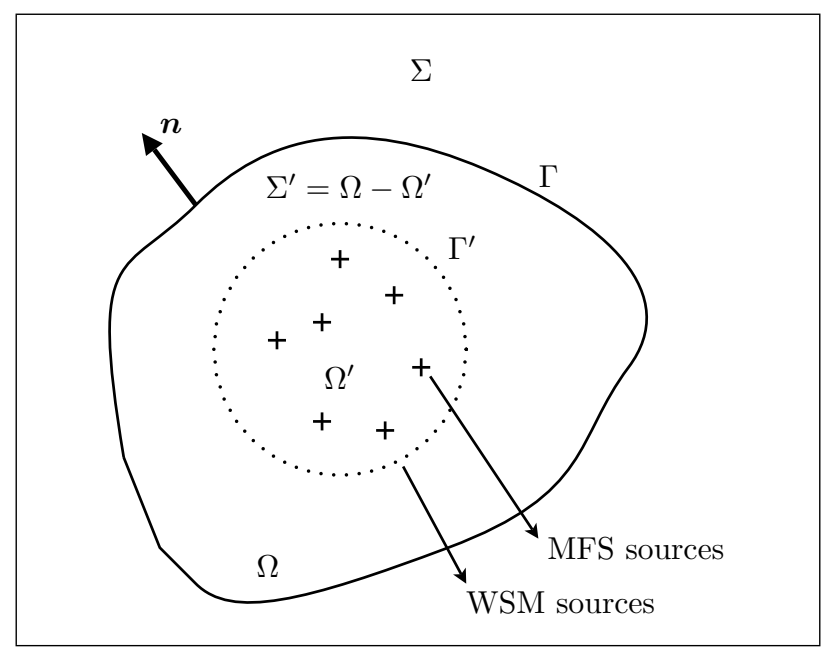

Figure 1. Vibrating body and the virtual sources.

The single-layer potential seeks to represent $p$ as

$$
p(\boldsymbol{r})=\int_{\Gamma} \sigma\left(\boldsymbol{r}_{s}\right) G\left(\boldsymbol{r}, \boldsymbol{r}_{s}\right) d \Gamma_{\boldsymbol{r}_{s}} \quad \boldsymbol{r} \in \Sigma,
$$

where $G$ is the free-space Green's function and $\sigma$ is a density function on $\Gamma$. Applying the boundary condition of a given normal pressure on $\Gamma$, leads to the boundary integral equation

$$
-\frac{1}{2} \sigma(\boldsymbol{r})+\int_{\Gamma} \sigma\left(\boldsymbol{r}_{s}\right) \frac{\partial G\left(\boldsymbol{r}, \boldsymbol{r}_{s}\right)}{\partial \boldsymbol{n}} d \Gamma \boldsymbol{r}_{s}=\frac{\partial p(\boldsymbol{r})}{\partial \boldsymbol{n}} \quad \boldsymbol{r} \in \Gamma
$$

\subsection{The Wave Superposition Method}

The simple idea behind this method is that the acoustic field of a complex radiator (cf. Figure 1) can be reconstructed as a superposition of individual simple sources. The equivalency of the WSM to the Helmholtz integral formulation has been shown by Koopman [8], thus validating the superposition integral:

$p(\boldsymbol{r})=\mathrm{j} \omega \rho \int_{\Omega} q\left(\boldsymbol{r}_{s}\right) G\left(\boldsymbol{r}, \boldsymbol{r}_{s}\right) \mathrm{d} \Omega\left(\boldsymbol{r}_{s}\right) \quad \boldsymbol{r} \in \Gamma \cup \Sigma$,

$\rho$ is the density of the medium, $\omega$ is the angular frequency of the harmonic vibration of the surface $\Gamma$ defining the interior region $\Omega$. The source strength is denoted by $q\left(\boldsymbol{r}_{s}\right)$, evaluated at $\boldsymbol{r}_{s}$ inside $\Omega$.

To reduce equation (6) to a numerical form, it's convenient to assume the sources distributed on $\Gamma^{\prime}$ inside $\Omega$. If this surface is divided into $N$ sufficiently small elements, we can approximate the normal velocity on the surface of the radiator,

$$
u_{n}(\boldsymbol{r}) \approx \sum_{i=1}^{N} Q_{i} \frac{\partial G\left(\boldsymbol{r}, \boldsymbol{r}_{s}\right)}{\partial \boldsymbol{n}_{r}} \quad \boldsymbol{r} \in \Gamma \quad \boldsymbol{r}_{s} \in \Gamma^{\prime},
$$

where $Q_{i}$ is the volume velocity of the simple source. Since $u_{n}(\boldsymbol{r})$ is known, $Q_{i}$ is given in matrix form by

$$
Q=[D]^{-1} u_{n}
$$


After finding the source strength vector $Q$, the pressure field is calculated from

$$
p(\boldsymbol{r})=\mathrm{j} \omega \rho \sum_{i=1}^{N} G\left(\boldsymbol{r}, \boldsymbol{r}_{s}\right) Q_{i} \quad \boldsymbol{r} \in \Gamma \cup \Sigma \quad \boldsymbol{r}_{s} \in \Gamma^{\prime},
$$

thanks to the linearized Euler equation

$$
\mathrm{j} \omega \rho u_{n}(\boldsymbol{r})=\frac{\partial p(\boldsymbol{r})}{\partial \boldsymbol{n}_{r}} .
$$

While there is much scope for discussion on the number and location of the interior sources for optimum accuracy, the method is claimed to be superior to the usual boundary integral formulation of the exterior acoustic problem since the source and collocation points never coincide, there are no problems associated with singularity. However, the superposition integral exhibits non-uniqueness at critical wave numbers, revealed by the layer potential formulations of the WSM principle.

\subsection{The Method of Fundamental Solutions}

For this method, equation (6) is still satisfied, because the sources can be placed anywhere inside the radiator. So, on numerical aspect, the MFS is an another version of the WSM as the source intensities satisfy the same equation [22],

$$
u_{n}(\boldsymbol{r})=\sum_{i=1}^{M} A_{i} \frac{\partial G\left(\boldsymbol{r}, \boldsymbol{r}_{s^{\prime}}\right)}{\partial \boldsymbol{n}_{r}} \quad \boldsymbol{r} \in \Gamma \quad \boldsymbol{r}_{s^{\prime}} \in \Omega
$$

Here, a least-square functional of this equation can be minimized [9], to obtain the best positions for the fictitious sources providing the optimal solutions. Another optimal MFS positioning has been recently proposed using the image method [23].

The difference between these the WSM and the MFS approaches is that the randomly distributed sources lead to higher condition number, unsuitable for effective numerical calculation, but the MFS does not exhibit critical wave numbers, except for some special sources positioning [24]. Thus, and apart from these particular cases, this method cannot be written in terms of a layer potential because these sources do not respect the Helmholtz integral positioning requirements. However, the MFS allows the system of equation to be overdetermined by a greater number of collocation points.

\subsection{Non-uniqueness}

Considering the vibration cavity of the Figure 1, the three main types of the WSM established on a virtual surface $\Gamma^{\prime}$ placed in $\Omega$ (with interior region $\Omega^{\prime}$ and exterior region $\left.\Sigma^{\prime}\right)$, are as follows:

(1) Single-layer potential integral

$$
p(\boldsymbol{r})=\int_{\Gamma^{\prime}} \sigma\left(\boldsymbol{r}_{s}\right) G\left(\boldsymbol{r}, \boldsymbol{r}_{s}\right) \mathrm{d} \Gamma_{\boldsymbol{r}_{s}}^{\prime}, \quad \boldsymbol{r} \in \Gamma \cup \Sigma .
$$

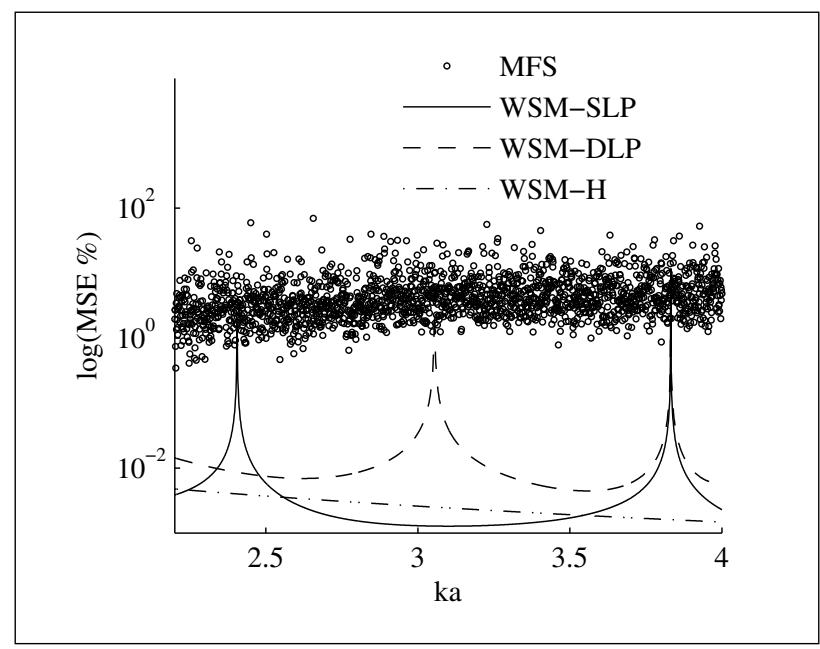

Figure 2. Critical wave numbers for three WSM formulations and the MFS.

(2) Double-layer potential integral

$$
\int_{\Gamma^{\prime}} \mu\left(\boldsymbol{r}_{s}\right) \frac{\partial G\left(\boldsymbol{r}, \boldsymbol{r}_{s}\right)}{\partial \boldsymbol{n}_{r_{s}}} \mathrm{~d} \Gamma^{\prime} \boldsymbol{r}_{s}= \begin{cases}p(\boldsymbol{r}), & \text { if } \boldsymbol{r} \in \Sigma \\ p(\boldsymbol{r})+\frac{\mu(\boldsymbol{r})}{2}, & \text { if } \boldsymbol{r} \in \Gamma\end{cases}
$$

(3) Hybrid combination

$$
\begin{gathered}
\int_{\Gamma^{\prime}} \gamma\left(\boldsymbol{r}_{s}\right)\left(G\left(\boldsymbol{r}, \boldsymbol{r}_{s}\right)+\alpha \frac{\partial G\left(\boldsymbol{r}, \boldsymbol{r}_{s}\right)}{\partial \boldsymbol{n}_{r_{s}}}\right) \mathrm{d} \Gamma_{\boldsymbol{r}_{s}}^{\prime} \\
= \begin{cases}p(\boldsymbol{r}), & \text { if } \boldsymbol{r} \in \Sigma \\
p(\boldsymbol{r})+\frac{\gamma(\boldsymbol{r})}{2}, & \text { if } \boldsymbol{r} \in \Gamma\end{cases}
\end{gathered}
$$

in which $G\left(\boldsymbol{r}, \boldsymbol{r}_{S}\right)$ is the free-space Green's function evaluated between the observation point and the source, located respectively at $\boldsymbol{r}$ and $\boldsymbol{r}_{s}$. On the virtual surface $\Gamma^{\prime}$, Green's theorem leads to the Helmholtz boundary integral equation for interior region and its differentiated form.

Considering the case of the Single Layer Potential formulation (WSM-SLP), Jeans [16] shows that if the excited wave number $k$ equals or is close to an interior Dirichlet eigenvalue, equation (12) has a non-unique solution. For the Double Layer Potential formulation (WSMDLP), non-uniqueness for equation (13) occurs at interior Neumann eigenvalues. The Hybrid combination (WSM$H$, equation 14) ensures a unique solution with an imaginary $\alpha$ because a real coefficient define an interior Robin eigenproblem.

Figure 2 shows the Mean Squared Error (MSE) obtained with the previous formulations and for an infinite cylinder (radius $R$ ). This estimator is defined as

$$
M S E=\sqrt{\frac{\sum_{i=1}^{N}\left|P 2_{i}-P 1_{i}\right|^{2}}{\sum_{i=1}^{N}\left|P 1_{i}\right|^{2}}},
$$

where $P 2$ is the reconstructed pressure and $P 1$ its known values. The velocity distribution on the surface is generated by a point source located at $1.1 a$ of the center 
$(a=0.1 R)$. This figure highlights the fictitious frequencies arising from the classic WSMs, and the solution obtained by the WSM-H and with the MFS where the sources $(N=M=100)$ are located randomly on the inscribed circle (radius $a$ ). These eigenfrequencies for single-layer potential formulation are such that

$$
J_{n}(k a)=0, \quad n=0,1,2, \ldots
$$

and for double-layer-potential

$$
J_{n}^{\prime}(k a)=0, \quad n=0,1,2, \ldots
$$

where $J$ is the Bessel function and $J^{\prime}$ its derivative. With the WSM approach, a direct determination of the sources strength is made, it thus differs from the minimization process usually employed in fundamental solutions determination.

\subsection{Combined Wave Superposition Method}

To achieve uniqueness, we propose to merge the rigorous formalism underlying the WSM with the ease of choice for MFS sources locations. From the numerical formulation of WSM and MFS, we can write

$$
\begin{gathered}
{\left[\begin{array}{cc}
{\left[D_{\mathrm{WSM}}\right]} & {\left[D_{\mathrm{MFS} \rightarrow \mathrm{WSM}}\right]} \\
{\left[D_{\mathrm{WSM} \rightarrow \mathrm{MFS}}\right]} & {\left[D_{\mathrm{MFS}}\right]}
\end{array}\right]\left[\begin{array}{l}
\{Q\} \\
\{A\}
\end{array}\right]} \\
=\left[\begin{array}{c}
\left\{u_{n}\right\}_{\mathrm{WSM}} \\
\left\{u_{n}\right\}_{\mathrm{MFS}}
\end{array}\right],
\end{gathered}
$$

where $\left[D_{W S M}\right]$ is the classic WSM dipole, [ $\left.D_{\mathrm{MFS}}\right]$ the MFS coefficient matrix, [ $D_{\text {MFS } \rightarrow \text { WSM }}$ the coefficient matrix between MFS sources and WSM collocation points and $\left[D_{\mathrm{WSM} \rightarrow \mathrm{MFS}}\right]$ the coefficient matrix between WSM sources and the MFS collocation points.

The basic idea here is to keep a sufficient but small MFS terms number because those sources (refereed for the rest of the paper as the complementary sources) are incorporated in WSM formulation only for achieving uniqueness at critical wave numbers and not for improving the global accuracy. The resulting formulation of equation (18) is named the Combined WSM (CWSM) as it combines MFS sources with the WSM formulation. The main difficulty with this method is, as for CHIEF, the selection of interior points away from the internal standing waves nodes of the corresponding Dirichlet problem. The step-by-step method proposed by Petrov [25] for the source coordinates calculation could be an efficient algorithm to solve this particular problem.

\section{Numerical investigations}

\subsection{Ill-conditionning and regularization}

Basic rank-revealing decompositions show the rankdeficient nature of numerical problems defined by superposition methods. For the previous defined problem, the Figure 3 shows that only the first singular values (zone I)

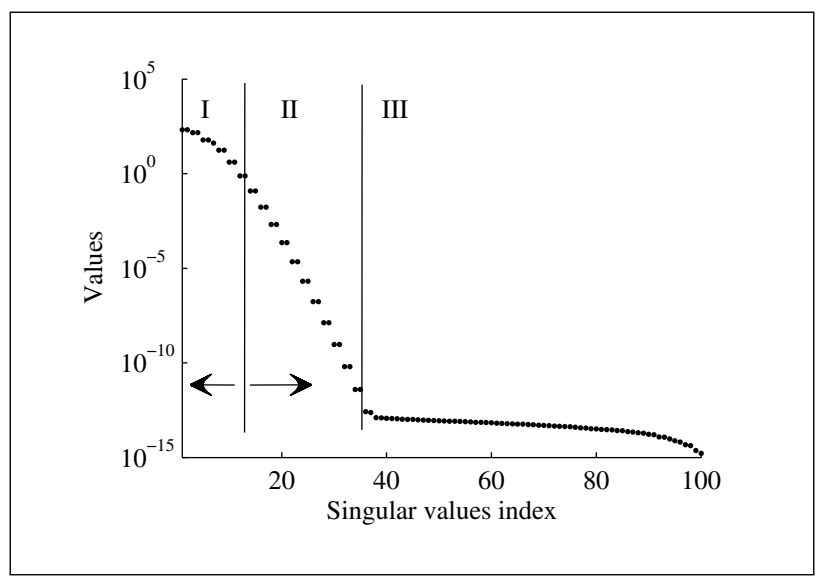

Figure 3. Singular Value Decomposition of influence matrix.

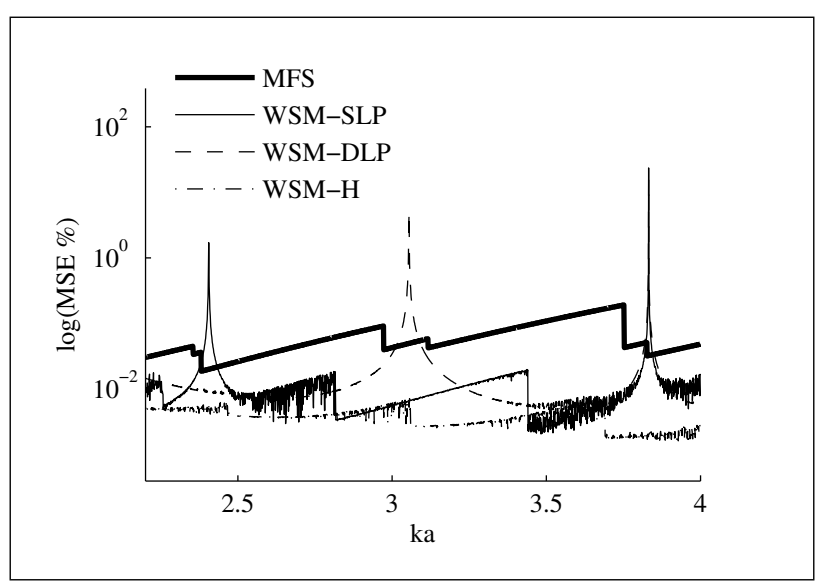

Figure 4. Truncated SVD influence on precision.

of the influence matrix ([D] in equation 8) are clearly relevant while additional values improved slightly the results (zone II) and others add numerical noise (zone III).

Thus, one common way to improve sources methods is to perform a Truncated SVD [26]. Applied to the formulations previously presented, results of Figure 4, with a factor of $10^{4}$ between the max and min singular values taken into account, exhibit a real improvement for the MFS but can be of consequence for the WSM results, without improving the solutions at eigenfrequencies. It must be remembered here that these critical wave numbers have nothing to do with ill-conditioning but are due to the nature of WSM method. Nevertheless, the number of singular value to take into account is not a trivial task, as optimal WSM solutions (Figure 2) are recover in this case with only two more singular values.

Other direct regularization methods like Tikhonov regularization have also been tested on superposition formulations, producing comparable or higher errors [19].

\subsection{Fictitious eigenfrequencies}

In order to demonstrate the accuracy of the proposed method (equation 18), the cylinder of the section 1.3 is reused, with 20 sources subtracted from the primary WSM 


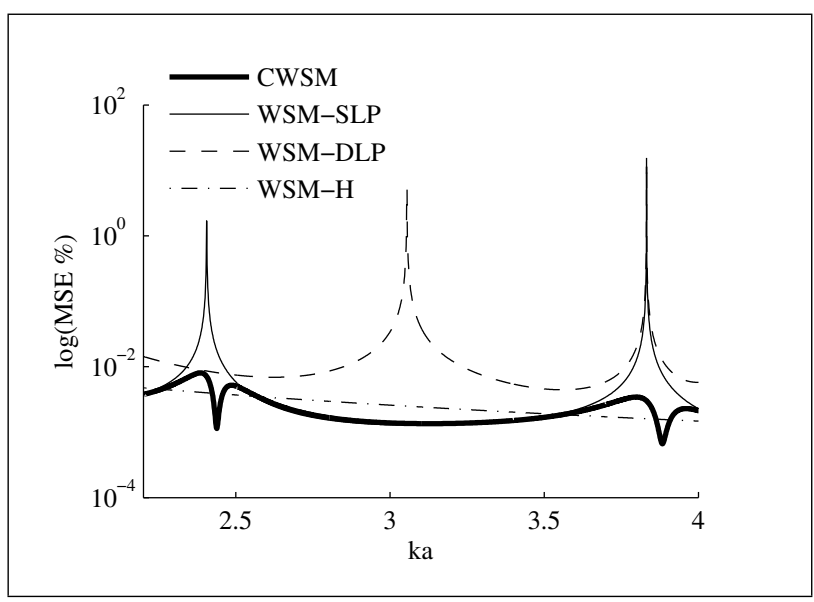

Figure 5. Complementary CWSM sources uniformly disposed.

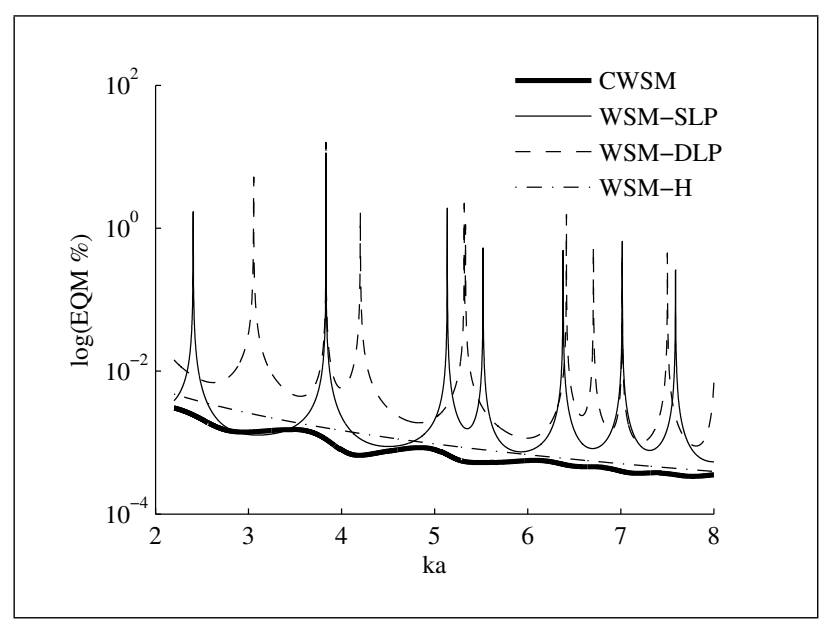

Figure 6. Random disposition of the complementary sources.

sources network $(N=80)$ and disposed on an inner circle of radius $b=0.95 a=0.095 R$ to form the complementary sources network $(M=20)$. Simple SVD inversion scheme is used to solve equation (18). Figure 5 shows the improvement brought by the CWSM (applied to the SLP form) at the critical wave numbers. However, with this configuration, the improvement is not optimal.

One efficient solution is to dispatch the complementary sources randomly, as any basic MFS do. Figure 6 highlights the benefits of this configuration for a wider band of frequencies. Here, the CWSM matches the hybrid formulation accuracy, while saving a third of the computation time [27].

Theoretically, it is sufficient to have a complementary source on a non-zero nodal line to overcome the uniqueness problem. But usually, this task is non-trivial because of non-regular geometry or while computing at high frequencies. As a consequence, the randomization of complementary sources appears to be good compromise between the computational cost of CWSM and its accuracy. Following the usual rule of discretization of four subdivisions per wavelength [27], experiments have shown satisfying

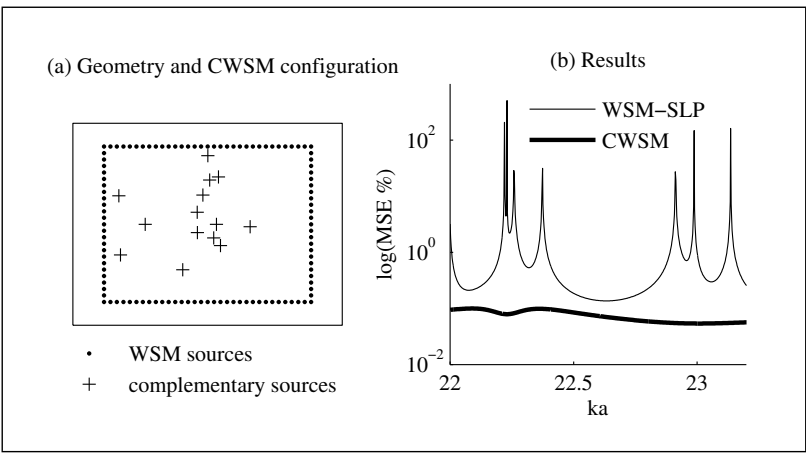

Figure 7. Reliability of the CWSM at high frequencies.

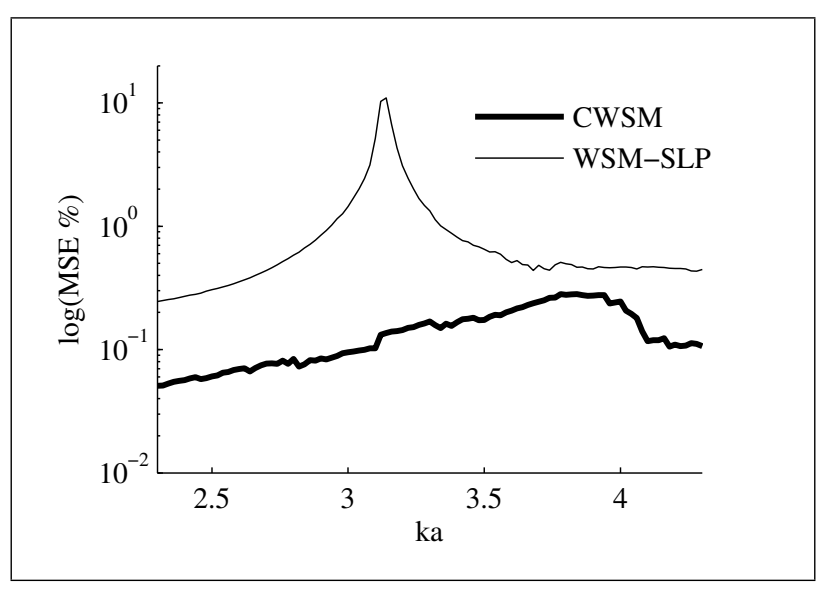

Figure 8. CWSM application on sphere.

results for a number of complementary sources approximately equal to a tenth of the WSM sources.

A last 2D application is performed on an infinite cylinder of rectangular section (cf. Figure 7a) to investigate the reliability of the CWSM at high frequencies.

Figure $7 \mathrm{~b}$ shows the results for $k a$ from 22 to 23.5, the CWSM performed as well as the previous cases. Thus, its accuracy depends solely of the classic frequency criterion, setting the maximum recommended distance between two WSM sources.

\subsection{D Application}

The method is now applied to a sphere of radius $R$. Superposition surface is set on a interior centered sphere of radius $a=0.1 R$. Figure 8 shows the MSE for the surface pressure reconstruction with classic WSM and with the CWSM, for a velocity distribution generated by a single point source at $1.1 a$ of the center. As for the planar examples, uniqueness of solutions is achieved with $M \approx N / 10$ internal sources at random position $(N=2450)$.

More sophisticated geometries and surface velocity distributions can be carried out using this approach. Nevertheless, as the classical WSM, the accuracy of the CWSM can be altered for geometries with corners (difficulties to take into account the discontinuity at tangent $\boldsymbol{n}$ ). 


\section{Conclusion}

The proposed combination of the wave superposition method and some internal sources provides a unique solution at any frequency with simple radiating monopoles. The computational cost for this Combined Wave Superposition Method is almost equivalent to the single layer potential formulation of the WSM. The positions of the superposition sources remain subject to the usual rules of thumb and the added source are preferentially placed at random positions. This distribution is the easiest way to avoid source placements at nodal lines, and to achieve uniqueness solutions for low to mid frequencies. As a consequence, if these nodal lines are known, the number of complementary sources can be optimized.

\section{References}

[1] H. A. Schenck: Improved integral formulation for acoustic radiation problems. J. Acoust. Soc. Am. 44 (1968) 41-58.

[2] A. J. Burton, G. F. Miller: The application of integral equation methods to the numerical solution of some exterior boundary-value problems. Proc. Roy. Soc. London, A 323 (1971) 201-210.

[3] D. S. Jones: Integral equations for the exterior acoustic problem. Q. J. Mech Appl. Math. XXVII (1974) 129-142.

[4] O. I. Panich: On the question of solvability of the exterior boundary value problems for the wave equation and Maxwell's equations. Russian Math. Surveys 20 (1965) 221-226.

[5] M. Guiggiani, G. Krishnasamy, T. J. Rudolphi, F. J. Rizzo: A general algorithm for the numerical solution of hypersingular boundary integral equations. J. Appl. Mech. 59 (1992) 604-614.

[6] J. J. Rêgo Silva, H. Power, L. C. Wrobel: A numerical implementation of a hypersingular boundary element method applied to 3D time-harmonic acoustic radiation problems. In: Boundary Elements XIV. Computational Mechanics Publ., Southampton and Elsevier, London, 1992, 271-287.

[7] B. Stupfel, A. Lavie, J.-N. Decarpigny: Combined integral equation and null-field method for the exterior acoustic problem. J. Acoust. Soc. Am. 83 (1988) 927-941.

[8] G. H. Koopmann, L. Song, J. B. Fahnline: A method for computing acoustic fields based on the principle of wave superposition. J. Acoust. Soc. Am. 86 (1989) 2433-2438.

[9] P. S. Kondapalli, D. J. Shippy, G. Fairweather: Analysis of acoustic scattering in fluids and solids by the method of fundamental solutions. J. Acoust. Soc. Am. 91 (1992) 1844-1854.

[10] R. Kress, A. Moshen, B. Brosowski: On the simulation source technique for exterior problems in acoustics. Math. Methods Appl. Sci. 8 (1986) 585-597.

[11] S. A. Yang: A boundary integral equation method using auxiliary interior surface approach for acoustic radiation and scattering in two dimensions. J. Acoust. Soc. Am. 112 (2002) 1307-1317.

[12] M. E. Johnson, S. J. Elliott, K.-H. Baek, J. Garcia-Bonito: An equivalent source technique for calculating the sound field inside an enclosure containing scattering objects. J. Acoust. Soc. Am. 104(1998) 1221-1231.

[13] M. B. S. Magalhaes, R. A. Tenebaum: Sound sources reconstruction techniques: a review of their evolution and new trends. Acta Acustica united with Acustica 90 (2004) 199-220.

[14] L. Song, G. H. Koopmann, J. B. Fahnline: Numerical errors associated with the method of superposition for computing acoustic fields. J. Acoust. Soc. Am. 89 (1991) 2625-2633.

[15] D. T. Wilton, I. C. Mathews, R. A. Jeans: A clarification of nonexistence problems with the superposition method. J. Acoust. Soc. Am. 94 (1993) 1676-1680.

[16] R. Jeans, I. C. Mathews: The wave superposition method as a robust technique for computıng acoustic helds. J. Acoust. Soc. Am. 92 (1992) 1156-1166.

[17] S. Marburg, S. Amini: Cat's eye radiation with boundary elements: comparative study on treatment of irregular frequencies. J. Comput. Acoust. 13 (2005) $21-45$.

[18] J.-Y. Hwang, S.-C. Chang: A retracted boundary integral equation for exterior acoustic problem with unique solution for all wave numbers. J. Acoust. Soc. Am. 90 (1991) 116/1180 .

[19] A. Sarkissian: Method of superposition applied to patch near-field acoustic holography. J. Acoust. Soc. Am. 118 (2005) 671-678.

[20] J. B. Fahnline, G. H. Koopmann: A numerical solution for the general radiation problem based on the combined methods of superposition and singular-value decomposition. J. Acoust. Soc. Am. 90 (1991) 2808-2819.

[21] X. Yu, H. Yuying, M. Xiaoqiang: Wave superposition method based on virtual source boundary with complex radius vector for solving acoustic radiation problems. Acta Mech. Solida Sin. 17 (2004) 12-19.

[22] G. Fairweather, A. Karageorghis, P. A. Martin: The method of fundamental solutions for scattering and radiation probTems. Eng. Anal. Boundary Elem. 27 (2003) 759-769.

[23] J. T. Chen, Y. T. Lee, S. R. Yu, S. C. Shieh: Equivalence between Trefftz method and method of fundamental solution for the annular Green's function using the addition theorem and image concept. Eng. Anal. Boundary Elem. 33 (2009) $678-688$

[24] I. L. Chen: Using the method of fundamental solutions in conjunction with the degenerate kernel in cylindrical acoustic problems. J. Chin. Inst. Eng. 29 (2006) 445-457.

[25] A. J. Petrov: An adaptation of auxiliary sources method for stationary acoustic problems. Wave Motion 39 (2004) 169180.

[26] C. Hansen: The truncated SVD as a method for regularization. BIT 27 (1987) 534-553.

[27] W. Benthien, A. Schenck: Nonexistence and nonuniqueness problems associated with integral equation methods in acoustics. Comput. Struct. 65 (1997) 295-305. 